\title{
Direct load control by distributed imperialist competitive algorithm
}

\author{
Fengji LUO, Junhua ZHAO (ه), Haiming WANG, \\ Xiaojiao TONG, Yingying CHEN, Zhao Yang DONG
}

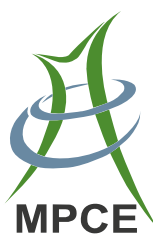

\begin{abstract}
Demand side management techniques have drawn significant attentions along with the development of smart grid. This paper proposes a new direct load control (DLC) model for scheduling interruptible air conditioner loads. The model is coordinated with the unit commitment and economic dispatch to minimize the total operation cost over the whole dispatch horizon. The network constraints are also considered in the model. To ensure the thermal comfort of the occupants, we are among the first to incorporate the advanced two-parameter thermal inertia dynamical model of customer houses into the DLC model to calculate the indoor temperature variation. This paper also proposes a distributed imperialist competitive algorithm to effectively solve the model. The simulation studies prove the efficiency of the proposed methodology.
\end{abstract}

Keywords Direct load control, Imperialist competitive algorithm, Demand side management

\section{Introduction}

With the increasing penetration of renewable energy sources and ever-increasing load demand, power system is experiencing the transition from a state "where relatively

CrossCheck date: 14 October 2014

Received: 31 July 2012/Accepted: 2 November 2012/Published online: 17 December 2014

(C) The Author(s) 2014. This article is published with open access at Springerlink.com

F. LUO, J. ZHAO, H. WANG, Y. CHEN, Centre for Intelligent Electricity Networks (CIEN), The University of Newcastle, Callaghan, NSW 2308, Australia

$(\bowtie)$ e-mail: andy.zhao@newcastle.edu.au

X. TONG, The Hunan First Normal University, Changsha, China

Z. Y. DONG, The University of Sydney, Sydney, NSW 2006, Australia well behaved demand is matched with well predictable generation" to a state where both demand and generation sides are becoming increasingly time-varying and stochastic [1]. This transition encourages the adoption of demand side management to re-shape the load profiles. The most commonly used demand side management approach is known as the direct load control (DLC), which aims to schedule the cycling of the customers' controllable appliances to achieve a certain operation objective.

In the summer days, air-conditioners are large energy consumers. Thus, designing suitable methods to schedule the cycling of air conditioner loads (ACLs) can effectively curtain the peak load in summer days. Since the primary usage of air-conditioners is to provide thermal comfort for customers, the most important constraint of ACL scheduling is obviously minimizing customers' thermal discomfort. Besides, DLC scheduling should be coordinated with the existing power system operation tasks such as unit commitment (UC) and economic dispatch (ED).

In the literature, many efforts have been done in DLC. In [2], the authors integrated interruptible load management (ILM) into DLC to provide ancillary services; in [3], the authors proposed a dynamical optimal power flow model to online select the interruptible loads while incorporating the network constraints; in [4], the authors reported three interruptible load management programs of Taiwan power company; in [5], the authors used the iterative deepening genetic algorithm to perform the DLC scheduling; a DLC based unit commitment model to minimize the system production cost is presented in [6]; the authors in [7] proposed an optimal power flow based framework for the independent system operator (ISO) to real-time select the interruptible load offers; the authors in [8] used probabilistic methods to do distributed interruptible load shedding; the authors in [9] proposed a reliabilityconstrained unit commitment model by integrating 
probabilistic spinning reserve and interruptible load. Literature [10] adopted a multi-pass dynamic programming to schedule the ACLs such that both of the generating costs and peak load reduction are minimized over the whole dispatch period; literature [11] proposed a unit commitment model incorporating large air conditioner loads, and used the fuzzy dynamic programming method to solve it; the authors in [12] designed a fuzzy logic based controller which recognizes several important customer preferences and desires. Authors in [13] presented a direct load control model in virtual power plant (VPP) operation, and used commercial software to simulate the thermal transition process of different buildings.

By reviewing the literature, we found that most of the previous works are based on the assumption that the scheduled ACL groups are homogeneous, which means that ACL groups are assumed to have the identical building environments and the customers have the same thermal preferences. However, in practice, the heterogeneous building environment will cause different indoor temperature variations, and different people may have different thermal preferences. In order to minimize customers' thermal discomfort, a more accurate model is needed to depict the thermal transition process of the building while considering the customers' different thermal preferences. Furthermore, to the best of the authors' knowledge, the existing DLC methods have not taken the transmission system capability constraints into account. Based on the above considerations, the main contributions of this paper are highlighted as follows.

- Propose a novel DLC model by taking the transmission network constraints into account;

- Among the first to incorporate a comprehensive thermal inertia model into DLC scheduling to satisfy customers' different thermal preferences.

- Propose a new distributed ICA algorithm to effectively solve the proposed DLC model.

This paper is organized as follows. In section 2, proposed DLC model is introduced; in section 3, the thermal inertia model is introduced; in section 4, proposed distributed imperialist competitive algorithm is introduced; approach to solve the model is presented in section 5; case studies are given in section 6; finally, conclusions are drawn in section 7 .

\section{DLC model formulation}

\subsection{Objective function}

The DLC model aims to minimize the total system operation costs over the dispatch horizon. The objective function is formulated as (1). $\min F=\sum_{t=1}^{T}\left(C_{g e n}^{t}+C_{i l}^{t}\right)$

where $t$ is the time interval index and $T$ is the total number of the time intervals; $C_{g e n}^{t}$ and $C_{i l}^{t}$ represent the generation cost and the ACL interruption cost at time interval $t$, respectively. We assume that the utility provides the discounted retail price for the customers who participate in the DLC program. Then $C_{i l}^{t}$ can be represented by the profit loss of the utility by promoting the DLC program. $C_{g e n}^{t}$ and $C_{i l}^{t}$ are calculated as (2) and (3).

$$
\begin{aligned}
& C_{g e n}^{t}=\sum_{G=1}^{G}\left[\left(F C\left(P_{g}^{t}\right)+S C_{g}^{t} \cdot\left(1-u_{g}^{t-1}\right)\right) \cdot u_{g}^{t}\right] \\
& C_{i l}^{t}=\sum_{n=1}^{N}\left(p r \cdot(1-\eta) \cdot C P_{n} \cdot s_{n}^{t}+p r \cdot\left(1-s_{n}^{t}\right) \cdot C P_{n}\right)
\end{aligned}
$$

where $g$ and $G$ represent the generator unit index and the total number of the units, respectively; $n$ and $N$ denote the index and the total number of the ACL groups, respectively; $u_{g}^{t}$ represents the state of the $g$ th unit at $t: 0$ OFF and $1-\mathrm{ON} ; s_{n}^{t}$ represents the $n$th ACL group at $t: 0$ OFF and 1-ON; $P_{g}^{t}$ is the active power output of the unit $g$ at time $t(\mathrm{~kW}) ; F C\left(P_{g}^{t}\right)$ is the fuel cost function of the units $(\$) ; S C_{g}^{t}$ is the startup cost of the unit $g$ at time $t(\$) ; \eta$ is the discount rate of the retailed electricity price provided by the utility (\%);CP $P_{n}$ is the capacity of the $n$th ACL group $(\mathrm{kW}) ; p r$ is the retailing electricity price $(\$ / \mathrm{kWh}) . F C\left(P_{g}^{t}\right)$ is with the form of (4) and $S C_{g}^{t}$ is represented by (5).

$F C_{g}^{t}=a_{g} \cdot\left(P_{g}^{t}\right)^{2}+b_{g} \cdot P_{g}^{t}+c_{g}$

$S C_{g}^{t}=\left\{\begin{array}{c}H S C_{g}, \text { if } M D T_{g} \leq T G_{g}^{o f f} \leq M D T_{g}+C S H_{g} \\ C S C_{g}, \text { if } \quad T G_{g}^{o f f}>M D T_{g}+C S H_{g}\end{array}\right.$

where $a_{g}, b_{g}$, and $c_{g}$ are fuel cost coefficients of unit $g$; $H S C_{g}$ and $C S C_{g}$ are the hot and cold startup costs of unit $g$, respectively (\$); $T G_{g}^{o n}$ and $T G_{g}^{o f f}$ denote the Duration during which the unit $g$ is continuously ON and OFF (hours); $M D T_{g}$ is the minimum down time of unit $g$; $\mathrm{CSH}_{g}$ is the cold startup hour of the unit $g$ (hours).

\subsection{Constraints}

1) Load balance constraint

$\sum_{g=1}^{G} P_{g}^{t}=P L^{t}-I L^{t}$

$I L^{t}=\sum_{n=1}^{N} C P_{n} \cdot\left(1-s_{n}^{t}\right)$ 
where $P L^{t}$ is the forecasted system load at time interval $t(\mathrm{~kW}) ; I L^{t}$ is the total interrupted load capacity at time $t(\mathrm{~kW})$.

2) Generator power output constraint

$P_{g}^{\min } \leq P_{g}^{t} \leq P_{g}^{\max }$

where $P_{g}^{\min }$ and $P_{g}^{\max }$ are the minimum and maximum power limits $(\mathrm{kW})$ of unit $g$.

3) Generator ramp rate constraint

$R_{\text {down }, g} \leq P_{g}^{t}-P_{g}^{t-1} \leq R_{\text {up }, g}$

where $R_{\text {down,g }}$ and $R_{u p, g}$ are ramp up and down rate of unit $g$.

4) Generator minimum online/offline time constraint

$\left\{\begin{array}{l}T G_{g}^{o n} \geq G M U T_{g} \\ T G_{g}^{o f f} \geq G M D T_{g}\end{array}\right.$

where $G M U T_{g}$ and $G M D T_{g}$ are the minimum offline time (hours) and online time (hours) of unit $g$.

5) Network power flow constraint

$$
\left\{\begin{array}{c}
P_{i}^{t}=P L_{i}^{t}-R L_{i}^{t}+P B^{t}+U_{i}^{t} \sum_{j=1}^{I} U_{j}^{t}\left(G_{i j} \cos \theta_{i j}^{t}+B_{i j} \sin \theta_{i j}^{t}\right) \\
Q_{i}^{t}=Q L_{i}^{t}+U_{i}^{t} \sum_{j=1}^{I} U_{j}^{t}\left(G_{i j} \cos \theta_{i j}^{t}-B_{i j} \sin \theta_{i j}^{t}\right)
\end{array}\right.
$$

where $i$ is the bus index; $I$ is the set of the buses; $L$ is the set of the transmission lines; $P_{i}^{t}$ and $Q_{i}^{t}$ are the active and reactive power of bus $i$ at time interval $t(\mathrm{~kW}) ; P L_{i}^{t}$ and $Q L_{i}^{t}$ are the active and reactive load on bus $i$ at time $t(\mathrm{~kW}) ; P B_{i}^{t}$ is the payback energy of bus $i$ at time $t(\mathrm{~kW}) ; R L_{i}^{t}$ is the reduced load of bus $i$ at time $t(\mathrm{~kW}) ; U_{i}^{t}$ is the voltage magnitude of bus $i$ at time $t ; \theta_{i j}^{t}$ is the phase angle deviation of branch $i j$ at time $t ; G_{i j}$ and $B_{i j}$ are real part and imaginary part of the nodal admittance matrix.

6) Bus voltage constraint

$U_{i}^{\min } \leq U_{i}^{t} \leq U_{i}^{\max }$

where $U_{i}^{\min }$ and $U_{i}^{\max }$ are the minimum and maximum voltage magnitude of bus $i$.

7) Apparent power constraints for transmission lines

$S_{i j}^{t} \leq S_{i j}^{\max }$

where $S_{i j}^{t}$ is the apparent power of branch $i j$ at time $t(\mathrm{~kW})$; and $S_{i j}^{\max }$ is the maximum apparent power of branch $i j$ $(\mathrm{kW})$.

$S_{i j}^{\max }(t)$ is calculated as

$S_{i j}^{t} \sqrt{\left(P_{i j}^{t}\right)^{2}+\left(Q_{i j}^{t}\right)^{2}}$

$$
\begin{aligned}
P_{i j}^{t} & =\left(U_{i}^{t}\right)^{2} G_{i j}-U_{i}^{t} U_{j}^{t}\left(G_{i j} \cos \theta_{i j}^{t}+B_{i j} \sin \theta_{i j}^{t}\right) \\
Q_{i j}^{t} & =-\left(U_{i}^{t}\right)^{2} B_{i j}-U_{i}^{t} U_{j}^{t}\left(G_{i j} \cos \theta_{i j}^{t}-B_{i j} \sin \theta_{i j}^{t}\right)
\end{aligned}
$$

8) ACL minimum online time constraint. To avoid frequently switch on/off and protect the mechanism equipment of the ACLs, the ACL groups are constrained by (18).

$T A_{n}^{o n} \geq A M U T_{n}$

where $T A_{n}^{o n}$ is the duration during which the ACL group $n$ is continuously ON (hours); and $A M U T_{n}$ is the minimum online time of ACL group $n$ (hours);

9) Indoor temperature dead band. The indoor temperature must be constrained by the temperature dead band, which refects different customers' preferences.

$T P \_L o w_{n}^{i n} \leq T P_{n}^{i n}(t) \leq T P \_U p_{n}^{i n}$

where $T P_{n}^{i n}(t)$ is the indoor temperature of ACL group $n$ at time $t ; T P_{-} L_{o w} w_{n}^{i n}(t)$ and $T P_{-} L_{o w} w_{n}^{i n}(t)$ are the lower and upper limits of comfort temperature range of ACL group $n\left({ }^{\circ} \mathrm{C}\right)$.

To calculate the indoor temperature profiles of the rooms in each ACL group after switching off the ACs, the thermal inertia model introduced in next section is applied.

\section{Thermal appliances modeling}

\subsection{Thermostatically load modeling}

The key to ensure the occupants' thermal comfort is to fully understand and model the dynamic thermal process of the buildings. In the smart house study, the one parameter thermal model has been widely used in many literatures [14-16], which is shown in Fig. 1a. The one-parameter model takes into account of parameters like internal and external temperatures, but only considers the thermal resistance of walls and neglects walls' thermal capacitance. In this paper, a more complex and accurate two-parameter is represented in Fig. 1b. The house is divided into two components, one of which is the internal of the house and the other is the additional thermal mass such as walls with much larger thermal capacitance.

However, the variation of indoor air temperature of a house could be considerably different when taking into account of thermal capacitance of walls [15]. This is due to the fact that the heat gain of a house can be divided into two parts: the relatively steady-state transmission resulting from temperatures differences between the indoor air and outdoor surroundings, and the unsteady-state gain due to the varying intensity of solar radiation on the walls. The unsteady-state heat flow across walls is very complicated 


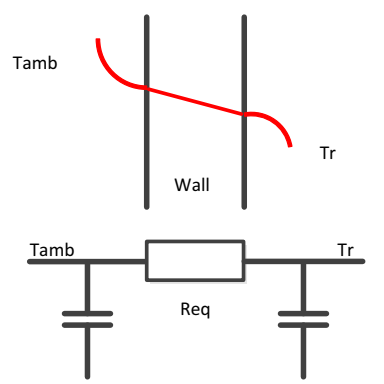

(a)

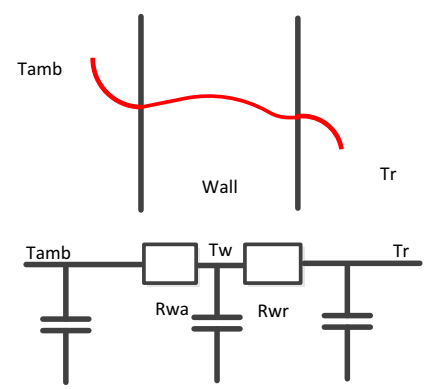

(b)
Fig. 1 a One parameter model. b Two parameter model

as part of heat passing through walls is captured and later released to either the indoor air or outdoor ambient. Therefore, the thermal dynamic model of a two-parameter model could be expressed as below [17].

$$
\begin{aligned}
& \frac{\mathrm{d} T_{r}(t)}{\mathrm{d} t}=\frac{1}{M_{a} \times C p_{a}} \\
& \times\left(\frac{\mathrm{d} Q_{g a i n \_a}(t)}{\mathrm{d} t}-\frac{\mathrm{d} Q_{e x \_w \_r}(t)}{\mathrm{d} t}-\frac{\mathrm{d} Q_{a c}(t)}{\mathrm{d} t}\right) \\
& \frac{\mathrm{d} T_{w}(t)}{\mathrm{d} t}=\frac{1}{M_{w} \times C p_{w}} \times\left(\frac{\mathrm{d} Q_{\text {gain_w }}(t)}{\mathrm{d} t}+\frac{\mathrm{d} Q_{e x \_w \_r}(t)}{\mathrm{d} t}\right) \\
& \frac{\mathrm{d} Q_{\text {gain_a }}(t)}{\mathrm{d} t}=\frac{T_{a m b}-T_{r}}{R_{e q}} \\
& \frac{\mathrm{d} Q_{e x \_w \_r}(t)}{\mathrm{d} t}=\frac{T_{w}-T_{r}}{R_{w r}} \\
& \frac{\mathrm{d} Q_{a c}(t)}{\mathrm{d} t}=C O P \times P_{a c} \\
& \frac{\mathrm{d} Q_{g a i n \_w}(t)}{\mathrm{d} t}=\frac{T_{a m b}-T_{w}}{R_{w a}}
\end{aligned}
$$

where $T_{r}$ is the room temperature $\left({ }^{\circ} \mathrm{C}\right) ; T_{w}$ is the wall temperature $\left({ }^{\circ} \mathrm{C}\right) ; M_{a}$ and $M_{w}$ are the mass of air inside the house and the walls (kg); $C p_{a}$ and $C p_{w}$ are the heat capacities of the air and the wall $\left(\mathrm{J} / \mathrm{kg}^{*} \mathrm{k}^{-1}\right) ; Q_{\text {gain_a }}$ is the heat gain by the indoor air from the ambient $(\mathrm{J}) ; Q_{\text {gain_w }}$ is the heat gain by the wall from the ambient (J); $Q_{a c}$ is the cooling energy delivered by air conditioner (J); COP is the coefficient of performance of air conditioner; $Q_{e \__{\_} w_{r} r}$ is the heat exchange between the wall and indoor air (J); $R_{e q}$ is the equivalent thermal resistance of the house envelope $\left(\mathrm{m}^{2} \mathrm{~K} / \mathrm{W}\right) ; T_{a m b}$ is the ambient temperature $\left({ }^{\circ} \mathrm{C}\right) ; R_{w r}$ is the thermal resistance between the wall inner surface and the indoor air $\left(\mathrm{m}^{2} \mathrm{~K} / \mathrm{W}\right) ; R_{w a}$ is the thermal resistance between the wall outer surface and the ambient $\left(\mathrm{m}^{2} \mathrm{~K} / \mathrm{W}\right) ; P_{a c}$ is the power of the individual airconditioner $(\mathrm{kW})$.
One of our previous studies [18] has proved that different complexities of models can pose significant impacts on the accuracy of cooling energy calculation, it is therefore decided that the complex thermal model is chosen in order to obtain more accurate results.

\subsection{Linearization of the thermal inertia model}

The thermal dynamic model in (19)-(24) can be linearized for convenient calculating the indoor temperature variation. For each dispatch time interval, $\Delta t$ is divided into $K$ steps. Provided that $K$ is large enough, we can assume that the temperatures of the ambient, walls, and the indoor air within any time step are constant. Hence, the change in temperatures can therefore be presented by the temperature difference between two adjacent time steps. Therefore, the thermal dynamic model can be linearized as (25)-(28).

$$
\begin{aligned}
& T P_{n}^{i n}(k)=\left(1-\frac{1}{M_{a} \times C p_{a} \times R_{e q}}\right) \times T_{r \_i n i t} \\
&+\frac{1}{M_{a} \times C p_{a} \times R_{e q}} \times T_{a m b \_i n i t} \\
&+\frac{T_{w \_i n i t}-T_{r \_ \text {init }}}{M_{a i r} \times C p_{a} \times R_{w r}}-S_{a c \_i n i t} \\
& \times \frac{Q_{a c}}{M_{a} \times C p_{a}}, k=1 \\
& T P_{n}^{i n}(k)=\left(1-\frac{1}{M_{a} \times C p_{a} \times R_{e q}}\right) \times T P_{n}^{i n}(k-1) \\
&+\frac{1}{M_{a} \times C p_{a} \times R_{e q}} \\
& \times T_{a m b}(k-1)+\frac{T_{w}(k-1)-T_{r}(k-1)}{M_{a} \times C p_{a} \times R_{w r}} \\
&-S_{a c}(k) \times \frac{Q_{a c}(k-1)}{M_{a} \times C p_{a}}, \forall k \in[2, K] \\
& T_{w}(k)=T_{w \_i n i t}+\frac{T_{a m b \_i n i t}-T_{w \_i n i t}}{M_{w} \times C p_{w} \times R_{w a}}+\frac{T_{r \_i n i t}-T_{w \_i n i t}}{M_{w} \times C p_{w} \times R_{w r}}, \\
& k=1 \\
& T_{w}(k)= T_{w}(k-1)+\frac{T_{a m b}(k-1)-T_{w}(k-1)}{M_{w} \times C p_{w} \times R_{w a}} \\
&+ \frac{T_{r}(k-1)-T_{w}(k-1)}{M_{w} \times C p_{w} \times R_{w r}}, \forall k \in[2, K]
\end{aligned}
$$

where $T_{\text {amb_init }}$ is the initial ambient temperature $\left({ }^{\circ} \mathrm{C}\right)$; $T_{w_{-} \text {init }}$ is the initial wall temperature $\left({ }^{\circ} \mathrm{C}\right) ; T_{r_{-} i n i t}$ is the initial room temperature $\left({ }^{\circ} \mathrm{C}\right)$.

\section{Distributed imperialist competitive algorithm}

The proposed DLC model is a binary, non-convex, high dimension, combinatorial optimization problem, which is hard to be handled by the conventional 
programming methods. There are many heuristic-based optimization algorithms that can be applied to solve the model, such as genetic algorithm (GA), particle swarm optimization (PSO) algorithm, differential evolutionary (DE) algorithm, etc. Recently, there is a new algorithm called imperialist competitive algorithm (ICA) [19] proposed has been applied to solve many industrial optimization problems [20-22]. A recent paper reported ICA is powerful to solve power system combinatorial problem [23]. As what will be shown in the later section of this paper, the evolution mechanism of ICA makes it be inherently suitable for paralleled implementation and its searching capability can thus be significantly improved. Therefore, in this paper we employ ICA to solve the proposed model. And in order to enhance its searching performance, we propose a distributed processing architecture for ICA.

\subsection{Imperialist competitive algorithm}

ICA mimics the competition among the imperialists. Each imperialist possess some colonies to form an empire, and tries to expanse its power by possessing the colonies of other empires. During the competition, weak empires collapse and powerful ones take possession of their colonies.

1) Empire initiation

As other heuristic searching algorithms, ICA maintains a population of individuals with the number of $N_{\text {pop }}$. In ICA, each individual is called a country. For a $N$-dimension minimization problem, the $i$ th country is a $1 \times N$ vector with the form of country $y_{i}=\left[x_{i}^{1}, x_{i}^{2}, \ldots, x_{i}^{N}\right]$. Each country represents a solution for a given problem, and its $\operatorname{cost} c_{i}$ can be obtained by evaluating it as $c_{i}=f\left(\right.$ country $\left._{i}\right)=f\left(\left[x_{i}^{1}\right.\right.$, $\left.x_{i}^{2}, \ldots, x_{i}^{N}\right]$ ).

In the start, $N_{\text {imp }}$ of the countries are selected as imperialists. The other $N_{p o p}-N_{\text {imp }}$ countries act as the colonies and are assigned to the $N_{i m p}$ imperialists in proportional to the powers of the imperialists. To calculate the imperialist powers, the cost of an imperialist (denoted as $c_{n}$ ) is firstly normalized,

$C_{n}=c_{n}-\max _{i}\left\{c_{i}\right\}$

where $C_{n}$ is the normalized cost. Then the normalized power of $n$th imperialist can be calculated by (30).

$p_{n}=\left|\frac{C_{n}}{\sum_{i=1}^{N} C_{i}}\right|$

The initial number of the colonies possessed by each imperialist $\left(N C_{n}\right)$ is in proportional to its power,

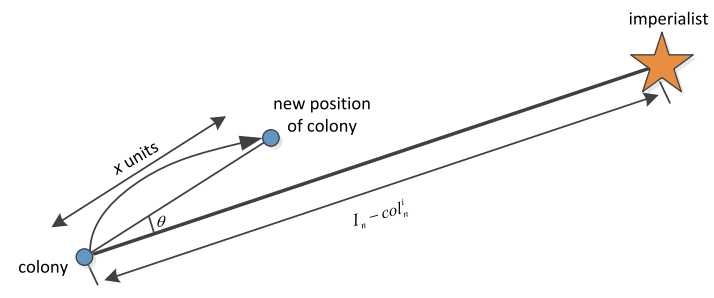

Fig. 2 Movement of the colony [19]

$N C_{n}=\operatorname{round}\left\{p_{n} \cdot N_{c o l}\right\}$

where and $N_{c o l}$ is the total number of the colonies. Then, $N C_{n}$ colonies are randomly selected and assigned to $n$th imperialist.

2) Moving the colonies towards the imperialists

As other heuristic searching algorithms, ICA has an iterative process to mutate the individuals. In each iteration, each colony moves moved toward the relevant imperialist by $x$ units,

$x=\omega \cdot\left(I_{n}-\operatorname{col}_{n}^{i}\right)$

where $\operatorname{col}_{n}^{i}$ and $I_{n}$ are the positions of $i$ th colony and imperialist of $n$th empire, respectively; $\omega$ is the weight factor which is usually set as a uniform random number within $(0,2)$, so as to make the colony move towards the imperialist in both sides. A random angle $\theta$ is also added as the deviation.

$\theta \sim U(-\gamma, \gamma)$

where $\gamma$ is the control parameter that adjusts the deviation from the original movement direction. Generally, the movement of the colony can be depicted by Fig. 2 .

3) Updating the positions of imperialist and a colony

After the movement, a colony may reach to a position with lower cost than the imperialist. In this case, the colony and the imperialist must exchange positions. The rest colonies of this empire should move forward the new imperialist position.

4) Imperialist competition

In each iteration, all empires compete with each other to try to take possession of colonies of other empires. The competition is based on the empires' powers. The total power of $n$th empire (denoted as $T C_{n}$ ) is calculated by (34).

$T C_{n}=c_{n}^{i m p}+\xi \cdot \frac{\sum_{i=1}^{N C_{n}} c_{n}^{i}}{N C_{n}}$

where $c_{n}^{i m p}$ is the cost of the imperialist of $n$th empire; $N C_{n}$ is the number $n$th empire's colonies; $c_{n}^{i}$ is the cost of the $i$ th colony of $n$th empire; $\xi$ is the weight factor. The normalized total power (denoted as $N T C_{n}$ ) and possession probability (denoted as $p_{p_{n}}$ ) of $n$th empire is represented as below. 
Table 1 Procedure of the distributed ICA

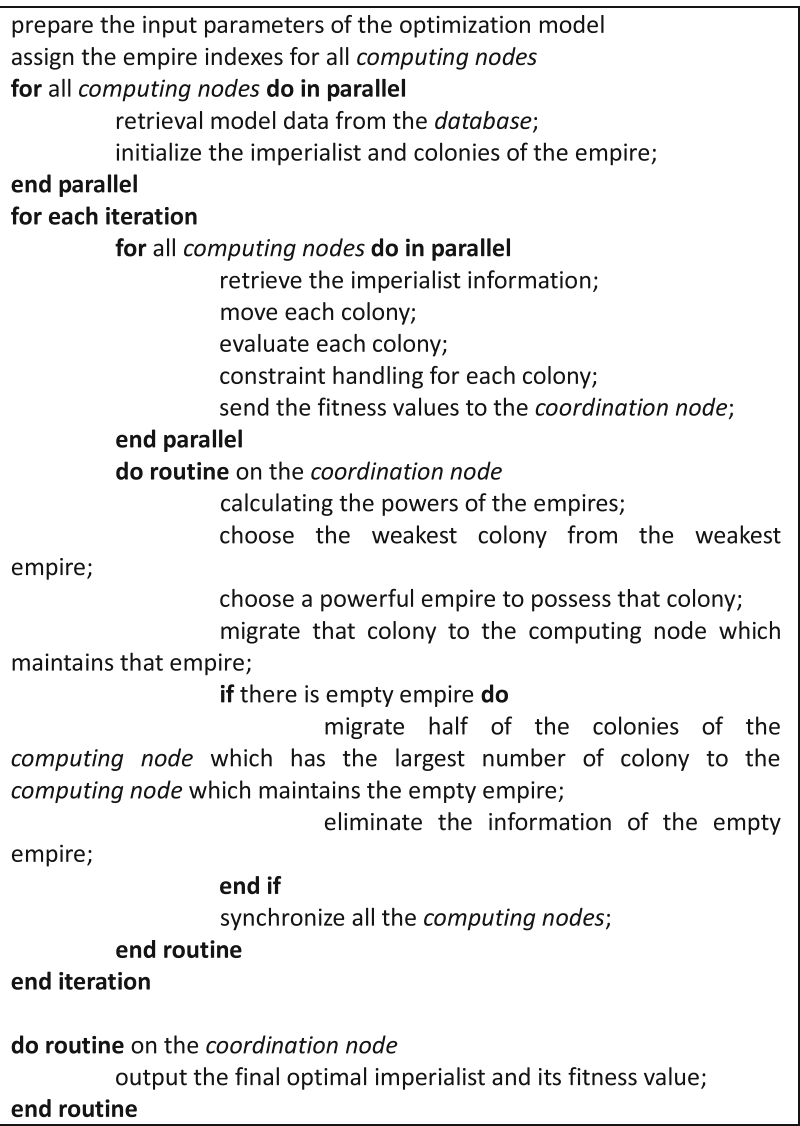

$N T C_{n}=T C_{n}-\max _{i}\left\{T C_{i}\right\}$

$p_{p_{n}}=\left|\frac{N T C_{n}}{\mid \sum_{i=1}^{N_{i m p}} N T C_{i}}\right|$

To divide the mentioned colony among empires based on their possession probability, vector $\boldsymbol{P}$ is formed as $\boldsymbol{P}=$ $\left[p_{p_{1}}, p_{p_{2}}, \ldots, p_{p_{\text {inp }}}\right]$. Then the vector $\boldsymbol{R}$ is formed as $\boldsymbol{R}=$ $\left[r_{r_{1}}, r_{r_{2}}, \ldots, r_{r_{i m p}}\right]$, with the elements are uniform distributed random numbers within $[0,1]$. Then vector $\boldsymbol{D}$ is formed by simply subtracting $\boldsymbol{R}$ from $\boldsymbol{P}$,

$\boldsymbol{D}=\boldsymbol{P}-\boldsymbol{R}=\left[p_{p_{1}}-r_{r_{1}}, p_{p_{2}}-r_{r_{2}}, \ldots, p_{p_{\text {imp }}}-r_{r_{\text {imp }}}\right]$

The mentioned colony then will be assigned to the empire whose relevant index in $\boldsymbol{D}$ is maximum. After each iteration, ICA checks whether there exists an empire which has lost all the colonies. If so, the empire will collapse and be eliminated.

5) Termination

The algorithm terminates when either of following two conditions is satisfied.

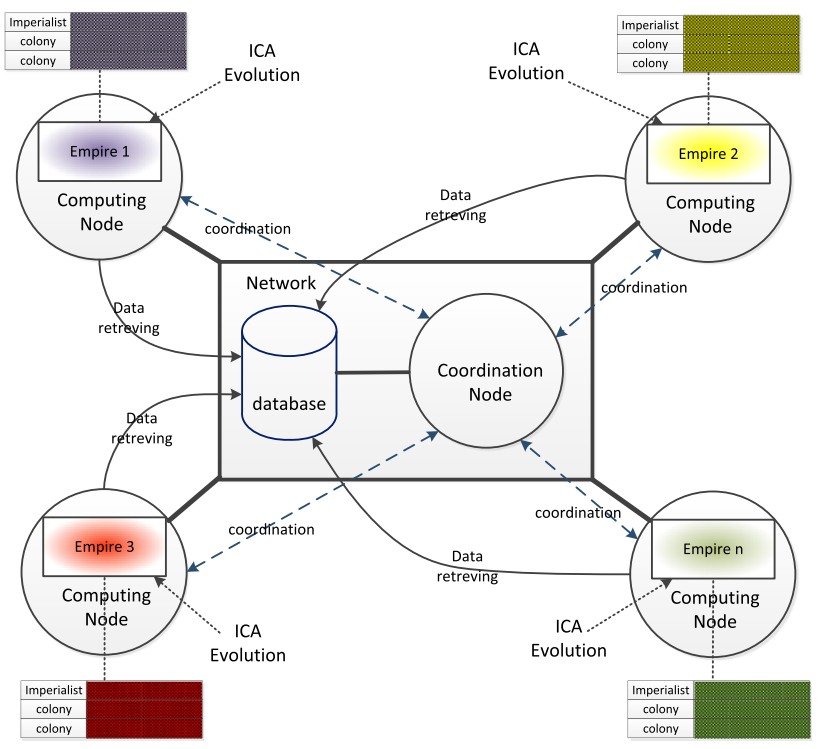

Fig. 3 Distributed processing architecture of the ICA

- All the empires collapse except for the most powerful one, and all the colonies have the same cost with the imperialist.

- The preset maximum iteration number is reached.

\subsection{Distributed processing architecture of ICA}

In ICA, multiple empires are formed and evolved separately. The communications among the empires occurs at the end of each iteration, where the weakest colony is reassigned and the empty empire is eliminated. This characteristic makes ICA suitable for parallel and distributed processing in nature. In this paper, we propose a distributed computing architecture for running ICA across networked processors, depicted in Fig. 3.

As depicted by Fig. 3, each computing node is assigned to maintain the countries of round $\left(N_{\text {emp }} / N_{\text {node }}\right)$ empires. The coordination node is responsible for coordinating the weakest colony re-assignment, empty empire eliminating, and statistics. A central database is established to store the input data of the optimization problem. The computing nodes retrieve data from the database through the network to perform the empire initialization, fitness evaluation and constraint handling.

With the evolution, the weakest empires are gradually eliminated. To balance the computing load, when $N_{\text {emp }}<N_{\text {node }}$, the idle computing nodes are assigned to part of the colonies of the empires which have largest number of colony. This is depicted by Fig. 4 .

The load balance algorithm is an active research topic in distributed computing. In future, more elaborate load balance strategy for the distributed ICA can be further studied. 


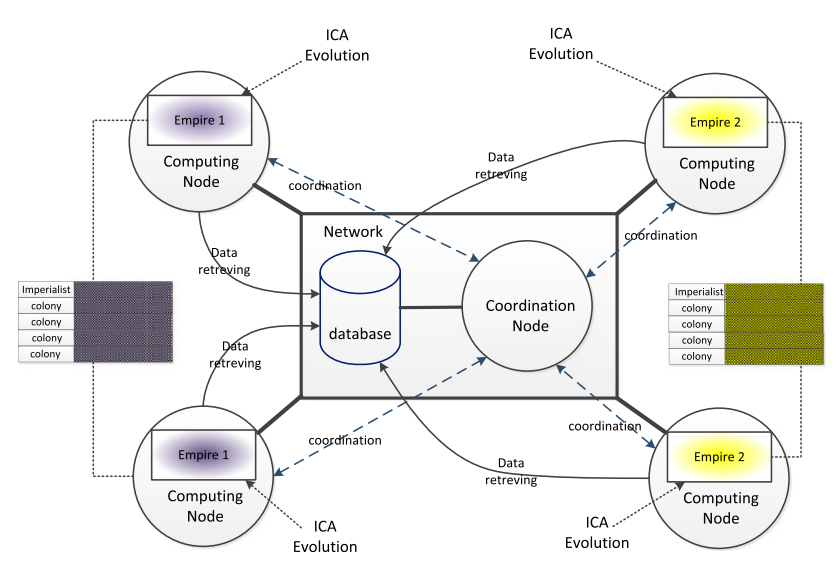

Fig. 4 Re-assignment of the computing nodes

The procedure of the distributed ICA is shown in Table 1. Firstly, the coordination node assigns the different empires to different computing nodes. Then all the computing nodes read the model data and do the initialization works in parallel. After that, all the computing nodes evolve their managed empires simultaneously. At the end of each iteration, the coordination node calculates the powers of all the empires, and mitigates some colonies from the compute node which maintains the most powerful empire to the compute node which maintains the weakest empire. After the evolution, the coordination nodes outputs the optimal solution.

\section{Distributed ICA based DLC approach}

By employing distributed ICA to solve the proposed model, the states of the generators and the ACL groups are encoded together as a country. The encoding of the population is shown as (38). For each country, the first $T * G$ dimensions represent the states of the generators on each UC time interval and the remaining $T_{d l c} * N$ dimensions represent the states of ACL groups on each DLC time interval. The symbol $T_{d l c}$ denotes the count of the DLC time intervals.

$$
\left[\frac{\frac{x_{1}^{1}\left|x_{1}^{2}\right| \ldots\left|x_{1}^{T * G}\right|\left|x_{1}^{T * G+1}\right| \ldots \mid x_{1}^{T * G+T_{d l c} * N}}{\frac{x_{2}^{1}\left|x_{2}^{2}\right| \ldots\left|x_{2}^{T * G}\right|\left|x_{2}^{T * G+1}\right| \ldots \mid x_{2}^{T * G+T_{d l c} * N}}{\vdots}}}{x_{N_{p o p}}^{1}\left|x_{N_{p o p}}^{2}\right| \ldots\left|x_{N_{p o p}}^{T * G}\right|\left|x_{N_{p o p}}^{T * G+1}\right| \ldots \mid x_{N_{p o p}}^{T * G+T_{d l c} * N}}\right]
$$

For each country, a certain constraint handling strategy is necessary to make it feasible. For a given country, following ACL group constraint handling procedures are firstly applied.

(1) On each DLC time interval, for each ACL group, if its state is OFF, then check whether the minimum online time constraint is satisfied. If not, then change its state to be $\mathrm{ON}$.

(2) On each DLC time interval, for each ACL group, calculate the indoor temperature variation based on the thermal inertia model. If its state is OFF and the calculated indoor temperature beyond the upper limit, then change its state to be $\mathrm{ON}$.

After handling the constraints of ACL groups, the generator constraint handling algorithm described in [24] is applied for the generators. After that, if the solution is still feasible, then the power flow calculation is performed to check whether the network constraints are satisfied. If so, then the solution is feasible; otherwise, the solution is marked as infeasible. The whole workflow is shown in Fig. 5.

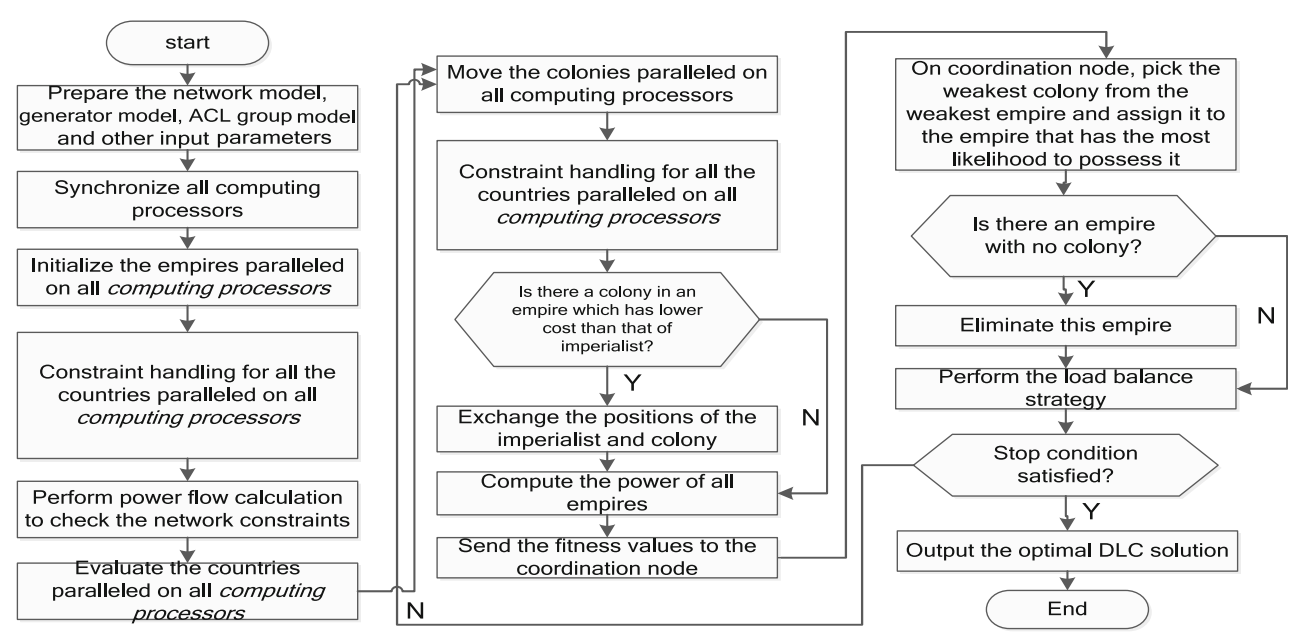

Fig. 5 Workflow of the distributed ICA based DLC 


\section{Simulation study}

\subsection{Distributed computing test bench}

We aggregate $6 \mathrm{PCs}$ and 1 workstation to establish a distributed computing test bench shown in Fig. 6. All of

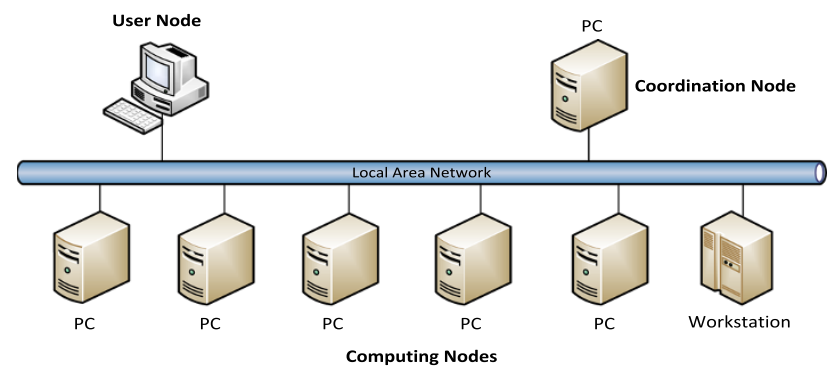

Fig. 6 Topology of the distributed computing test bench

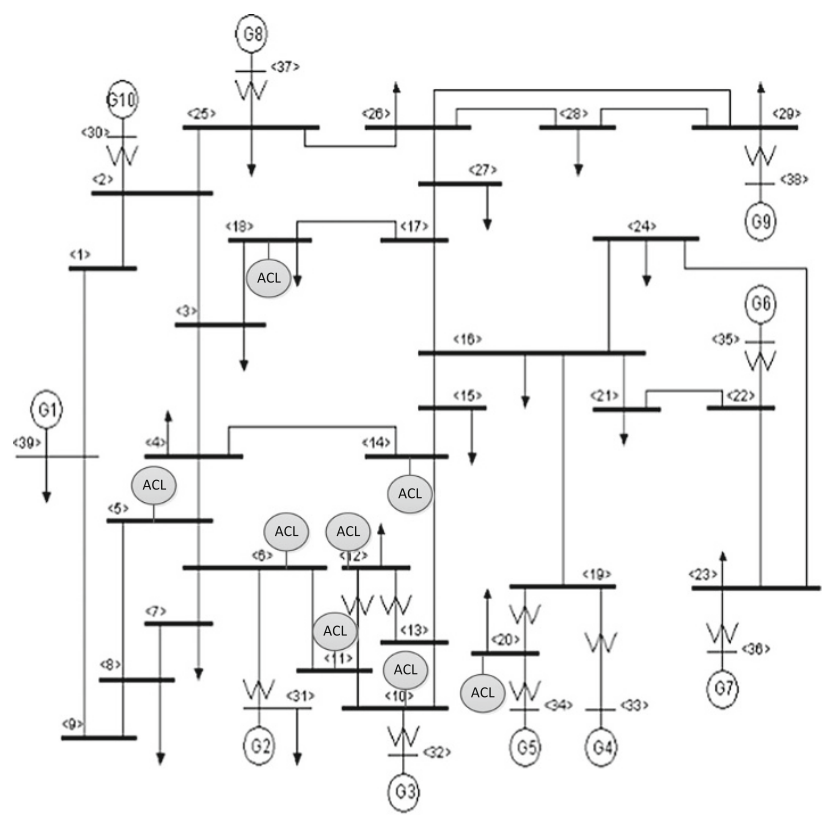

Fig. 7 The modified IEEE 39-bus system the 6 PCs are DELL 64-bit, 4-core, and with Intel ${ }^{\circledR}$ Core ${ }^{\mathrm{TM}}$ i5-2400 CPU and 4 Giga-byte RAM; the workstation is DELL 64-bit, 8-core, and with Intel ${ }^{\circledR}$ Core $^{\mathrm{TM}}$ i52400 CPU and 8 Giga-byte RAM. The operation systems of the 4 PCs are windows 7 while that of the workstation is windows server 2000. The 7 machines are located in different places and connected by local area network (LAN). One PC is selected as the coordination node and the others act as the computing nodes.

\subsection{Programing platform}

The MPICH2 [25] is used to implement the distributed ICA, with the $\mathrm{C}++$ programming language. $\mathrm{MPICH} 2$ is a high performance and portable implementation of the message passing interface (MPI) standard, and has been applied in many high performance computing applications. The power flow calculation is implemented by the interior point method. The development environment is Microsoft Visual Studio 2010.

\subsection{Experiment setup}

The IEEE 39-bus benchmark system [26] is used to test the proposed method. The system consists of 10 units and 29 branches. 8 ACL groups are set up on the bus 5, 6, 10, $11,12,14,18$, and 20. The ACL groups are assumed to have different building environments and different customer comfort preferences. The system is shown in Fig. 7. The information of the 6 ACL groups is shown in Table 2.

Table 3 ICA parameter setting

\begin{tabular}{ll}
\hline Population Size & 6000 \\
Maximum Iteration Time & 600 \\
$N_{\text {imp }}$ & 6 \\
$\gamma$ & $\pi / 4$ \\
\hline
\end{tabular}

Table 2 ACL group information

\begin{tabular}{|c|c|c|c|c|c|c|c|c|c|c|}
\hline $\begin{array}{l}\mathrm{ACL} \\
\text { group }\end{array}$ & $\begin{array}{l}\text { Total } \\
\text { capacity/ } \\
\text { MW }\end{array}$ & $\begin{array}{l}\text { Capacity of } \\
\text { each } \mathrm{AC} / \mathrm{kW}\end{array}$ & $\begin{array}{l}\text { Building } \\
\text { material }\end{array}$ & $\begin{array}{l}\text { Building } \\
\text { length } / \mathrm{m}\end{array}$ & $\begin{array}{l}\text { Building } \\
\text { width/m }\end{array}$ & $\begin{array}{l}\text { Building } \\
\text { height } / \mathrm{m}\end{array}$ & $\begin{array}{l}\text { Wall } \\
\text { thickness/ } \\
\text { m }\end{array}$ & $\begin{array}{l}\text { Minimum } \\
\text { online time/ } \\
\text { min }\end{array}$ & $\begin{array}{l}T P_{-} L o w_{n}^{i n} / \\
{ }^{\circ} \mathrm{C}\end{array}$ & $\begin{array}{l}T P \_U p_{n}^{i n} \\
{ }^{\circ} \mathrm{C}\end{array}$ \\
\hline 1 & 20 & 6 & Brick & 20 & 10 & 12 & 0.24 & 45 & 24 & 27 \\
\hline 2 & 40 & 4 & Brick & 18 & 12 & 10 & 0.3 & 30 & 24 & 28 \\
\hline 3 & 20 & 2 & Brick & 14 & 10 & 10 & 0.24 & 30 & 25 & 28 \\
\hline 4 & 10 & 4 & Wood & 18 & 10 & 8 & 0.3 & 30 & 22 & 26 \\
\hline 5 & 40 & 4 & Wood & 16 & 15 & 10 & 0.4 & 45 & 24 & 28 \\
\hline 6 & 20 & 6 & Wood & 20 & 15 & 12 & 0.35 & 45 & 26 & 28 \\
\hline 7 & 20 & 4 & Concrete & 18 & 12 & 11 & 0.4 & 30 & 22 & 27 \\
\hline 8 & 20 & 6 & Concrete & 19 & 15 & 12 & 0.3 & 30 & 22 & 26 \\
\hline
\end{tabular}



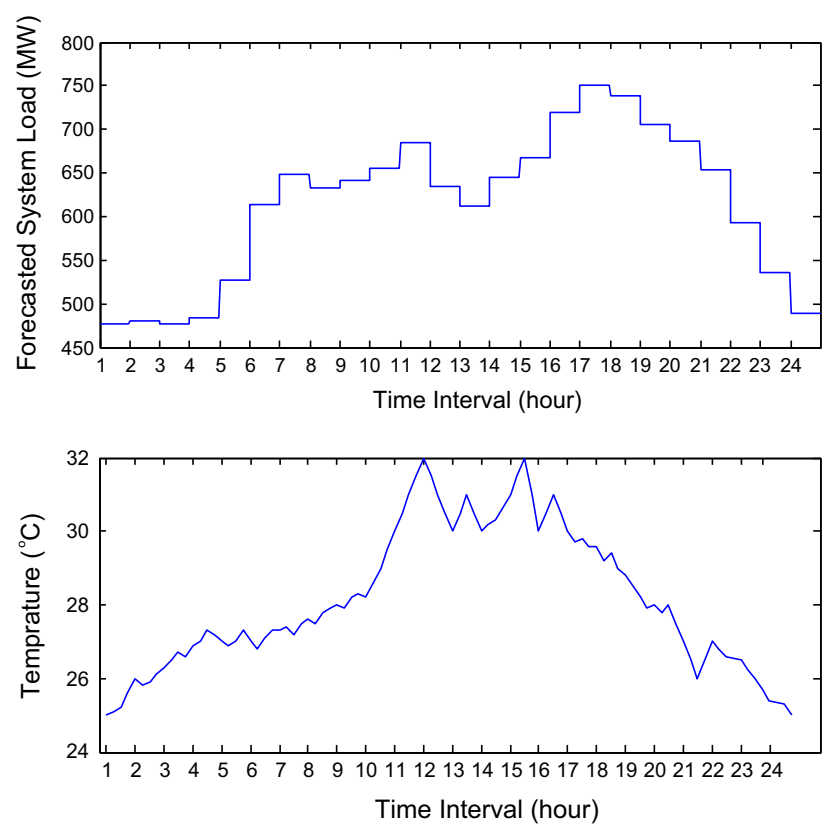

Fig. 8 Forecasted system load (up) and outdoor air temperature profile (down)

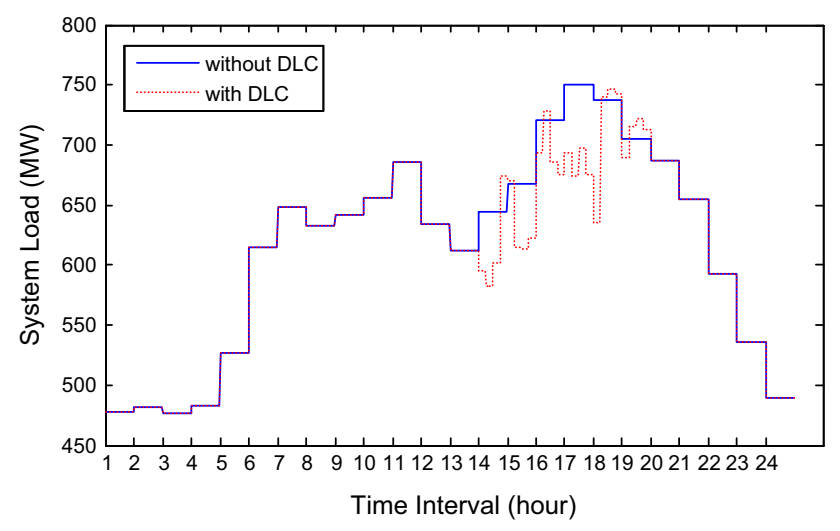

Fig. 9 Load patterns before and after the DLC

Table 4 System characteristics of the case studied

Total system operation cost with DLC (\$)

53927

Generation cost $(\$)$

49089

Load shedding cost $(\$)$

4838

Total shed load of the day (MWh)

285.25

Energy saving ratio (of interruptible capacity) (\%)

27.43

The generator data follows the literature [25]. The control parameters of the ICA are set as Table 3.

The 24-hour forecasted system load and outdoor air temperature profiles are shown as Fig. 8. It can be seen that the peak load occurs between 14:00 and 20:00, thus the DLC is executed during this period. The dispatch interval

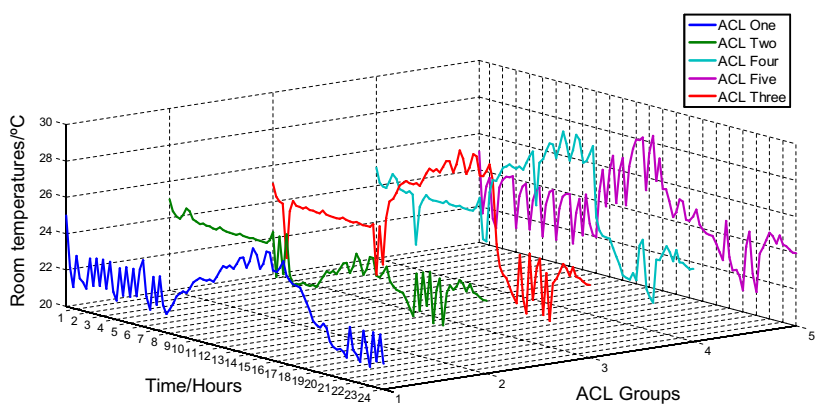

Fig. 10 Indoor temperature profile of the 5 ACL groups

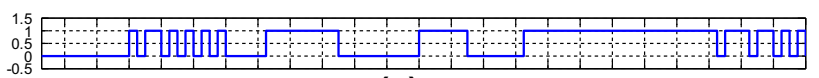

(a) ACL 1

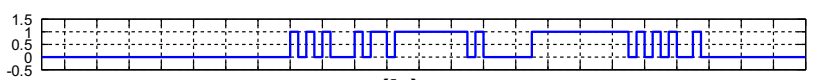

(b) ACL 2

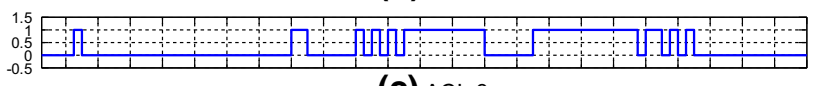

(c) ACL 3

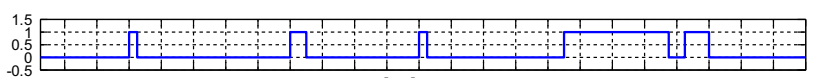

(d) ACL 4

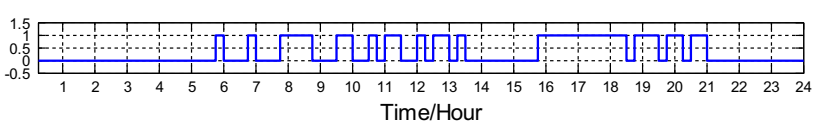

(e) ACL 5

Fig. 11 ON/OFF actions of the 5 ACL groups

is set to be 15 minutes. Thus there are totally 24 dispatch intervals.

\subsection{Numerical results}

The load patterns before and after the DLC scheduling are shown in Fig. 9. Table 4 reports the system characteristics of the case studied. The results show that the system peak loads are effectively shed by the DLC, with some secondary peak loads. Those secondary peak loads are caused by the resuming of the ACL groups which are switched off in previous time intervals. There are totally 285.25 MWh loads are curtained during the dispatch horizon (occupied $27.43 \%$ of the total ACL capacity), and the total operation cost is $\$ 53,927$.

The indoor temperature variations of the ACL groups are effectively controlled to ensure the customers' thermal comfort. For example, Fig. 10 shows the indoor temperature profile of 5 randomly selected ACL groups during the dispatch horizon. It can be clearly seen that the temperature is controlled within $\left[25^{\circ} \mathrm{C}, 28{ }^{\circ} \mathrm{C}\right]$. Figure 11 shows the scheduled ON/OFF states of the 5 ACL groups. 


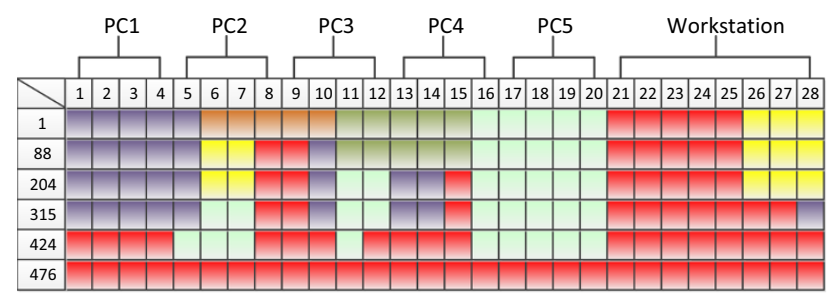

Empire $1 \square$ Empire $2 \square$ Empire $3 \square$ Empire $4 \square$ Empire $5 \square$ Empire 6

Fig. 12 Empire elimination process of the distributed ICA

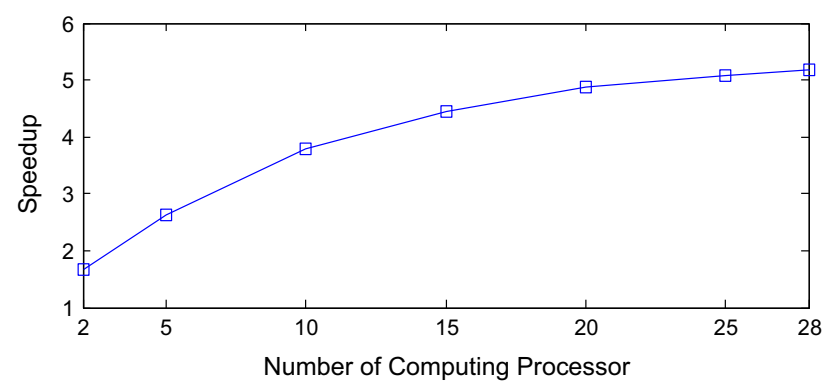

Fig. 13 Speedup of the distributed ICA

Figure 12 shows the empire migrating process of the distributed ICA. Figure 12 clearly shows that along with the elimination of the empires, the coordination node mitigates the colonies of the left empires among the processors to achieve the load balance. Finally, all the colonies of the only left empire (Empire 3) are maintained by all the processors.

Figure 13 reports the speedup achieved by increasing the number of the computing processors, relative to run the same case on a single process serially. In the single process, the total execution time of the simulation is 61,433 seconds. The results show that with the increase of processor number, the distributed ICA can significantly shorten the simulation time.

\section{Conclusions}

A comprehensive DLC model is proposed in this paper. The objective of the model is to schedule interruptible ACLs and generation units to minimize the total operation costs over the dispatch horizon, while taking the network constraints into account. An advanced two-parameter thermal inertia model is used to represent the thermal transition processes of buildings with heterogeneous characteristics.

A new distributed ICA algorithm is proposed to solve the DLC model. The distributed ICA is based on the ICA algorithm, and employs the process communication model to perform the distributed high performance searching. The proposed method is verified on the IEEE 39-bus benchmark system and a distributed computing test bed.

Acknowledgments This work was supported in part by National Natural Science Foundation of China (Key Project 71331001, General Project 71371065, 11171095, 71071025).

Open Access This article is distributed under the terms of the Creative Commons Attribution License which permits any use, distribution, and reproduction in any medium, provided the original author(s) and the source are credited.

\section{References}

[1] Ipakchi A, Albuyeh F (2009) Grid of the future. IEEE Power Energy Mag 7(2):52-62

[2] Huang KY, Huang YC (2004) Integrating direct load control with interruptible load management to provide instantaneous reserve for ancillary services. IEEE Trans Power Syst 19(3): $1626-1634$

[3] Majumdar S, Chattopadhyay D, Parikh J (1996) Interruptible load management using optimal power flow analysis. IEEE Trans Power Syst 11(2):715-720

[4] Chen CS, Leu JT (1990) Interruptible load control for Taiwan power company. IEEE Trans Power Syst 5(4):460-465

[5] Yao L, Chang WC, Yen RL (2005) An iterative deepening genetic algorithm for scheduling of direct load control. IEEE Trans Power Syst 20(3):1414-1421

[6] Hsu YY, Su CC (1991) Dispatch of direct load control using dynamic programming. IEEE Trans Power Syst 6(3):1056-1060

[7] Tuan LA, Bhattacharya K (2003) Competitive framework for procurement of interruptible load services. IEEE Trans Power Syst 18(2):889-897

[8] Argiento R, Faranda R, Pievatolo A et al (2012) Distributed interruptible load shedding and micro-generator dispatching to benefit system operations. IEEE Trans Power Syst 27(2): $840-848$

[9] Aminifar F, Fotuhi-Firuzabad M, Shahidehpour M (2009) Unit commitment with probabilistic spinning reserve and interruptible load considerations. IEEE Trans Power Syst 24(1):388-397

[10] Wei DC, Chen NM (1995) Air conditioner direct load control by multi-pass dynamic programming. IEEE Trans Power Syst 10(1):307-313

[11] Yang HT, Huang KY (1999) Direct load control using fuzzy dynamic programming. IEE Proc Gener Transm Distrib 146(3):294-300

[12] Salehfar H, Noll PJ, LaMeres BJ et al (1999) Fuzzy logic-based direct load control of residential electric water heaters and air conditioners recognizing customer preferences in a deregulated environment. In: Proceedings of the power engineering society summer meeting, vol 2. Edmonton, Canada, 18-22 Jul 1999, pp 1055-1060

[13] Ruiz N, Cobelo I, Oyazabal J (2009) A direct load control model for virtual power plant management. IEEE Trans Power Syst 24(2):959-966

[14] Hubert T, Grijalva S (2012) Modeling for residential electricity optimization in dynamic pricing environments. IEEE Trans Smart Grid 3(4):2224-2231

[15] Mortensen RE, Haggerty KP (1988) A stochastic computer model for heating and cooling loads. IEEE Trans Power Syst 3(3):1213-1219 
[16] Sansawatt T, Ochoa LF, Harrison GP (2012) Smart decentralized control of DG for voltage and thermal constraint management. IEEE Trans Power Syst 27(3):1637-1645

[17] Bălan R, Cooper J, Chao KM et al (2011) Parameter identification and model based predictive control of temperature inside a house. Energy Build 43(2/3):748-758

[18] Wang HM, Meng K, Luo FJ et al (2013) Demand response through smart home energy management using thermal inertia. In: Proceedings of the 2013 Australian universities power engineering conference (AUPEC'13), Hobart, Australia, 29 Sept-3 Oct 2013, 6 pp

[19] Atashpaz-Gargari E, Lucas C (2007) Imperialist competitive algorithm: an algorithm for optimization inspired by imperialistic competition. In: Proceedings of the IEEE congress on evolutionary computation (CEC'07), Singapore, 25-28 Sept 2007, pp 4661-4667

[20] Mohammadi-Ivatloo B, Rabiee A, Soroudi A et al (2012) Imperialist competitive algorithm for solving non-convex dynamic economic power dispatch. Energy 44(1):228-240

[21] Gargari E, Hashemzadeh F (2008) Colonial competitive algorithm: a novel approach for PID controller design in MIMO distillation column process. Int $\mathbf{J}$ Intell Comput Cybern 1(3):337-355

[22] Yousefi M, Darus AN, Mohammadi H (2012) An imperialist competitive algorithm for optimal design of plate-fin heat exchangers. Int J Heat Mass Trans 55(11/12):3178-3185

[23] Moghimi Hadji M, Vahidi B (2011) A solution to the unit commitment problem using imperialistic competition algorithm. IEEE Trans Power Syst 27(1):117-124

[24] Chung CY, Yu H, Wong KP (2011) An advanced quantuminspired evolutionary algorithm for unit commitment. IEEE Trans Power Syst 26(2):847-854

[25] Thakur R, Rabenseifner R, Gropp W (2005) Optimization of collective communication operations in MPICH. Int J High Perform Comput Appl 19(1):49-66

[26] Athay T, Padmore R, Virmani S (1979) A practical method for the direct analysis of transient stability. IEEE Trans Power Apparatus Syst 98(2):573-584

Fengji LUO is a post doctor associate of the Centre for Intelligent Electricity Networks (CIEN), The University of Newcastle, Australia. $\mathrm{He}$ obtained his bachelor and master degrees from Chongqing University, and doctor degree from the University of Newcastle. His research interests include smart grid planning, renewable energy dispatch, demand side management, and distributed computing.
Junhua ZHAO received his Ph.D. degree from the University of Queensland, Australia. Currently he is a senior lecturer at the University of Newcastle, Australia. His research interests include power system analysis and computation, smart grid, cyber physical system, electricity market, data mining and its applications.

Haiming WANG is a graduate of the School of Energy Science and Engineering, Central South University, China, and he received his Master of Science degree in New and Renewable Energy from Durham University, UK in 2010. He is now pursuing a PhD in The Centre for Intelligent Electricity Networks, the University of Newcastle. His research interests include thermostatically controlled loads and demand side management.

Xiaojiao TONG received the B.S. and M.S. degrees in mathematics from Wuhan University, Wuhan, China, and the Ph.D. degree in applied mathematics from Hunan University, Hunan, China, in 1983, 1986, and 2000, respectively. She has taught at Changsha University of Science and Technology, Hunan, China, and is now a professor and Vice President at Hunan First Normal University, Hunan, China. Her research interests include nonlinear optimization, stochastic programming and applications, power system analysis and power market.

Yingying CHEN is a research associate at the Centre for Intelligent Electricity Networks (CIEN), The University of Newcastle, Australia. She obtained her bachelor and master degrees from Chongqing University, and doctor degree from the University of Newcastle. Her research interests include wind power dispatch, wind farm planning and distributed computing.

Zhao YANG DONG is now Professor and Head of School of Electrical and Information Engineering, University of Sydney, Australia. He is immediate Ausgrid Chair Professor and Director of the Centre for Intelligent Electricity Networks (CIEN), the University of Newcastle, Australia. He previously held academic and industrial positions with the Hong Kong Polytechnic University, the University of Queensland, Australia and Transend Networks, Australia. His research interest includes smart grid, power system planning, power system security, load modeling, renewable energy systems, electricity market, and computational intelligence and its application in power engineering. He is an editor of IEEE Transactions on Smart Grid, and IEEE Power Engineering Letters. 